Homology, Homotopy and Applications, vol.21(1), 2019, pp.283-302

\title{
INTEGRAL COHOMOLOGY OF CONFIGURATION SPACES OF THE SPHERE
}

\section{CHRISTOPH SCHIESSL}

\author{
(communicated by Nathalie Wahl)
}

\begin{abstract}
We compute the cohomology of the unordered configuration spaces of the sphere $S^{2}$ with integral and with $\mathbb{Z} / p \mathbb{Z}$-coefficients using a cell complex due to Fox, Neuwirth, Fuks, Vainshtein and Napolitano.
\end{abstract}

\section{Contents}

1 Introduction $\quad 283$

1.1 Conventions . . . . . . . . . . . . . . . . . . 285

2 Configuration spaces of the plane $\quad 285$

2.1 Cellular decomposition of $C_{n}(\mathbb{C})^{+} \ldots \ldots \ldots . \ldots . \ldots . \ldots 285$

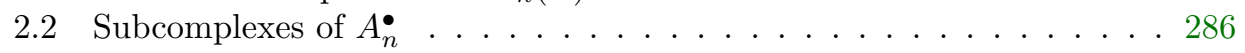

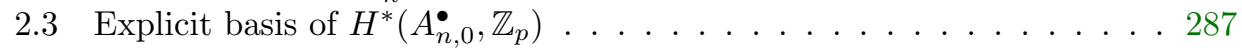

2.4 Bockstein homomorphisms . . . . . . . . . . . . . . . . . 288

3 Configuration spaces of the sphere 292

3.1 Cellular decomposition of $C_{n}\left(S^{2}\right)^{+} \ldots \ldots \ldots$. . . . . . . . 293

3.2 Mapping cone complex . . . . . . . . . . . . . . . . . . . . 293

4 Construction of (almost) a null homotopy 294

5 Proof of main theorem $\quad 297$

\section{Introduction}

For any topological space $X$, let

$$
F_{n}(X)=\left\{\left(x_{1}, \ldots, x_{n}\right) \in X^{n} \mid x_{i} \neq x_{j}\right\}
$$

be the ordered configuration space of $n$ distinct points in $X$. The symmetric group $S_{n}$ acts on $F_{n}(X)$ by permuting the points and the quotient

$$
C_{n}(X)=F_{n}(X) / S_{n}
$$

The author was supported by the grant ERC-2012-AdG-320368-MCSK.

This is part of the author's PhD thesis.

Received January 22, 2018, revised June 4, 2018, July 16, 2018; published on October 17, 2018.

2010 Mathematics Subject Classification: 55R80.

Key words and phrases: homology, configuration space.

Article available at http://dx.doi.org/10.4310/HHA.2019.v21.n1.a13

Copyright (C) 2018, Christoph Schiessl. Permission to copy for private use granted. 
is the unordered configuration space.

Despite their simple definition, getting a grasp of their topology is hard. The cohomology of configuration spaces has been widely studied (e.g., [8, 27, 22, 1, $10,24,9]$ ) but only few cases have been computed explicitly. Usually this is only possible if the space $X$ is very simple or one restricts to rational or $\bmod p$ coefficients. Aside from the Euclidean case due to Arnold [2], vanishingly few complete integral homology calculations are available.

On the other hand, the cohomology of many configuration spaces satisfies interesting properties, for example with rational coefficients homological stability [7] or eventual periodicity with $\bmod p$ coefficients $[17,5,16]$.

In this paper, we will completely compute $H^{*}\left(C_{n}\left(S^{2}\right), \mathbb{Z} / p \mathbb{Z}\right)$ and $H^{*}\left(C_{n}\left(S^{2}\right), \mathbb{Z}\right)$.

Theorem 1.1. Let

$$
B_{p}(n, r)=\left|\left\{\begin{array}{c|c}
1 \leqslant a_{1} \leqslant a_{2} \leqslant \cdots \leqslant a_{g} & 2 \sum_{i} p^{a_{i}}+2 \sum_{j} p^{b_{j}}-2 g-h=r \\
0 \leqslant b_{1}<b_{2}<\cdots<b_{h} & 2 \sum_{i} p^{a_{i}}+2 \sum_{j} p^{b_{j}} \leqslant n
\end{array}\right\}\right|
$$

and

$$
\left.B_{p}^{\prime}(n, r)=\mid \begin{array}{c|c}
1 \leqslant a_{1} \leqslant a_{2} \leqslant \cdots \leqslant a_{g} & 2 \sum_{i} p^{a_{i}}+2 \sum_{j} p^{b_{j}}+1-2 g-h=r \\
2 \sum_{i} p^{a_{i}}+2 \sum_{j} p^{b_{j}}+2 \leqslant n \\
1 \leqslant b_{1}<b_{2}<\cdots<b_{h} & p \nmid 2\left(n-2 \sum_{i} p^{a_{i}}-2 \sum_{j} p^{b_{j}}-1\right)
\end{array}\right\} \mid .
$$

Then

$$
\operatorname{dim} H^{r}\left(C_{n}\left(S^{2}\right), \mathbb{Z} / p \mathbb{Z}\right)=B_{p}(n, r)+B_{p}(n-1, r-2)-B_{p}^{\prime}(n, r)-B_{p}^{\prime}(n, r-1) .
$$

Corollary 1.2. We have

$$
\operatorname{dim} H^{r}\left(C_{n}\left(S^{2}\right), \mathbb{Z} / 2 \mathbb{Z}\right)=B_{2}(n, r)+B_{2}(n-1, r-2) .
$$

Our main tool is a cellular decomposition of $C_{n}\left(S^{2}\right)$ due to Napolitano [18]. It is an extension of the Fox-Neuwirth cell structure for $C_{n}\left(\mathbb{R}^{2}\right)[13]$ used by Fuks [14] and Vainshtein [25] to compute the $\bmod p$ cohomology of $C_{n}\left(\mathbb{R}^{2}\right)$.

Theorem 1.1 could also be deduced from [21, Th. 18.3]. However, our approach is more elementary and allows to determine the integral cohomology:

Theorem 1.3. The first cohomology groups $H^{r}\left(C_{n}\left(S_{2}\right), \mathbb{Z}\right)$ are

$$
\begin{array}{ll}
H^{0}\left(C_{n}\left(S_{2}\right), \mathbb{Z}\right)=\mathbb{Z}, & H^{1}\left(C_{n}\left(S_{2}\right), \mathbb{Z}\right)=0, \\
H^{2}\left(C_{n}\left(S_{2}\right), \mathbb{Z}\right)=\mathbb{Z} /(2 n-2) \mathbb{Z}, & H^{3}\left(C_{n}\left(S_{2}\right), \mathbb{Z}\right)= \begin{cases}0 & n=1,2, \\
\mathbb{Z} & n=3, \\
\mathbb{Z} \times \mathbb{Z} / 2 \mathbb{Z} & n \geqslant 4 .\end{cases}
\end{array}
$$

For $r \geqslant 4$, the cohomology groups $H^{r}\left(C_{n}\left(S^{2}\right), \mathbb{Z}\right)$ are finite and contain no elements of order $p^{2}$.

Hence we can reconstruct all integral cohomology groups by Theorem 1.1 and the universal coefficient Theorem. Previously, the cohomology of $C_{n}\left(S^{2}\right)$ was known with rational coefficients $[23,19,21]$, for low degree cases $[23,18]$, for mod 2 coefficients [4] and for mod $p$ coefficients [21]. The description of $H^{r}\left(C_{n}\left(S^{2}\right), \mathbb{Z}\right)$ seems to be new. 
We will first explain the computations of the cohomology of $C_{n}(\mathbb{C})$ with $\mathbb{Z} / p \mathbb{Z}$ coefficients by Fuks [14] and Vainshtein [25] and discuss the Fox-Neuwirth cell structure. Afterwards, we present the extension of this cell complex due to Napolitano [18] used to calculate $H^{*}\left(C_{n}\left(S^{2}\right), \mathbb{Z}\right)$ for $n \leqslant 9$. The main idea of this paper is the construction of a very specific chain homotopy that simplifies Napolitano's complex.

\subsection{Conventions}

We write

$$
\operatorname{Part}(n, s)=\left\{\left[n_{1}, \ldots, n_{s}\right] \in \mathbb{Z}_{>0}^{q} \mid n_{1}+\cdots+n_{s}=n\right\}
$$

for partitions of $n$ into $s$ positive summands, for example

$$
\operatorname{Part}(5,3)=\{[3,1,1],[1,3,1],[1,1,3],[2,2,1],[2,1,2],[1,2,2]\} .
$$

We call $s$ the length and $n$ the size of the partition.

The residue ring $\mathbb{Z} / m \mathbb{Z}$ is from now on abbreviated by $\mathbb{Z}_{m}$. For any abelian group $G$ and prime $p$, we write $G_{T_{p}}=\left\{g \in G \mid p^{n} g=0\right.$ for some $\left.n\right\}$ for the $p$-torsion subgroup.

\section{Acknowledgments}

I want to thank Frederick Cohen, Emanuele Delucchi, Emmanuel Kowalski, Paolo Salvatore, Johannes Schmitt, Junliang Shen for very helpful discussions and especially Rahul Pandharipande for his invaluable support.

\section{Configuration spaces of the plane}

\subsection{Cellular decomposition of $C_{n}(\mathbb{C})^{+}$}

The following construction comes from [14] and [25]. The projection

$$
\mathbb{C} \rightarrow \mathbb{R}, x+i y \mapsto x
$$

to the real line maps any configuration in $C_{n}(\mathbb{C})$ to a finite sets of points in $\mathbb{R}$. Counting the number of preimages of each of these points, we get a partition of $n$. Here we use that the one-dimensional line is ordered. The union of all points in $C_{n}(\mathbb{C})$ mapping to the same partition $n=n_{1}+\cdots+n_{s}$ and the point $\infty$ is an $n+s$ dimensional cell in the one point compactification $C_{n}(\mathbb{C})^{+}$. We denote this cell by $\left[n_{1}, \ldots, n_{s}\right]$. All such cells together with the point $\infty$ are a cellular decomposition of $C_{n}(\mathbb{C})^{+}$. Using Poincaré-Lefschetz duality for Borel-Moore homology [6], [26, Chapter $13.2]$

$$
H^{i}\left(C_{n}(\mathbb{C})\right)=\tilde{H}_{2 n-i}\left(C_{n}(\mathbb{C})^{+}\right),
$$

this cell complex can be used to compute the cohomology of $C_{n}(\mathbb{C})$.

The (co)-chains of the resulting (cochain)-complex $A_{n}^{\bullet}=\left(A_{n}^{r}\right)$ with the property

$$
H^{*}\left(C_{n}(\mathbb{C}), \mathbb{Z}\right)=H^{*}\left(A_{n}^{\bullet}\right)
$$

are the free $\mathbb{Z}$-modules

$$
A_{n}^{r}=\mathbb{Z} \operatorname{Part}(n, n-r) .
$$

The basis elements are the partitions $\left[n_{1}, \ldots, n_{s}\right] \in \operatorname{Part}(n, s)$ with $s=n-r$. The 
boundary maps $\delta: A_{n}^{r} \rightarrow A_{n}^{r+1}$ are

$$
\delta\left[n_{1}, \ldots, n_{s}\right]=\sum_{l=1}^{s-1}(-1)^{l-1} P\left(n_{l}, n_{l+1}\right)\left[n_{1}, \ldots, n_{l-1}, n_{l}+n_{l+1}, n_{l+2}, \ldots, n_{s}\right],
$$

with coefficients

$$
P(x, y)= \begin{cases}0 & \text { if } x \equiv y \equiv 1 \quad \bmod 2, \\
\left(\begin{array}{c}
\lfloor x / 2+y / 2\rfloor \\
\lfloor x / 2\rfloor
\end{array}\right) & \text { otherwise. }\end{cases}
$$

Intuitively, in the boundary of a cell, the points lying on two neighbouring vertical lines come together onto the same vertical line. The coefficient $P(x, y)$ gives a signed count of the different ways to combine two sets of $x$ and $y$ points on a line.

\subsection{Subcomplexes of $A_{n}^{\bullet}$}

As $P(x, y)=0$ for odd $x$ and $y$, the complex $A_{n}^{\bullet}$ can be written as a direct sum

$$
A_{n}^{\bullet}=A_{n, 0}^{\bullet} \oplus \cdots \oplus A_{n, n}^{\bullet}
$$

of subcomplexes $A_{n, t}^{\bullet}$ generated by partitions with $t$ odd entries.

Take any $I \subset\{1, \ldots, s+t\}$ with $t$ elements, say $I=\left\{i_{1}, \ldots, i_{t}\right\}$ where $i_{1}<\cdots<$ $i_{t}$. Then we insert $1^{\prime} s$ at the positions $i_{1}$ to $i_{t}$ with alternating signs:

$$
\operatorname{Ins}_{I}\left[a_{1}, \ldots, a_{s}\right]=(-1)^{\sum_{j} i_{j}}\left[a_{1}, \ldots, a_{i_{1}-1}, 1, a_{i_{1}}, \ldots, a_{i_{2}-2}, 1, a_{i_{2}-1}, \ldots\right] .
$$

The map

$$
\operatorname{Ins}_{t}=(-1)^{s t} \sum_{I \subset\{1, \ldots, s+t\},|I|=t} \operatorname{Ins}_{I}
$$

is actually a chain map

$$
\mathrm{Ins}_{t}: A_{n, 0}^{\bullet} \rightarrow A_{n+t, t}^{\bullet}
$$

that induces isomorphisms [25, Prop. 1]

$$
H^{r}\left(A_{n-t, 0}^{\bullet}\right) \simeq H^{r}\left(A_{n, t}^{\bullet}\right) .
$$

Hence we get

$$
H^{*}\left(A_{n}^{\bullet}\right)=H^{*}\left(A_{n, 0}^{\bullet}\right) \oplus H^{*}\left(A_{n-1,0}\right) \oplus \cdots \oplus H^{*}\left(A_{0,0}^{\bullet}\right) .
$$

As $A_{n, 0}^{r}=0$ if $n>2 r$, we can immediately deduce that the cohomology groups stabilize

$$
H^{r}\left(A_{n}^{\bullet}\right)=H^{r}\left(A_{n+1}^{\bullet}\right)
$$

if $n>2 r$. Later, we will use the notation

$$
H^{r}\left(C_{\infty}(\mathbb{C})\right)=H^{r}\left(C_{n}(\mathbb{C})\right)
$$

for any $n>2 r$.

Example 2.1. The cohomology group $H^{0}\left(C_{n}(\mathbb{C}), \mathbb{Z}\right)=\mathbb{Z}$ is generated by the class of

$$
(-1)^{n(n-1) / 2}[1, \ldots, 1]=\operatorname{Ins}_{n}([]) .
$$

For $n \geqslant 2$, the cohomology group $H^{1}\left(C_{n}(\mathbb{C}), \mathbb{Z}\right)=\mathbb{Z}$ is generated by the class of

$$
[2,1, \ldots, 1]-[1,2,1, \ldots, 1]+\cdots=(-1)^{(n-2)(n-3) / 2+n} \operatorname{Ins}_{n-2}[2] .
$$




\subsection{Explicit basis of $H^{*}\left(A_{n, 0}^{\bullet}, \mathbb{Z}_{p}\right)$}

We will now present the description of the group $H^{r}\left(A_{n, 0}^{\bullet}, \mathbb{Z}_{p}\right)$ by Vainshtein and work out some of the details and proofs omitted in [25].

Remark 2.2. In particular, the explicit formula for the base elements seems to be stated in a misleading way in [25] (definition of morphism $\Phi$, top of page 136). There, the operator Perm is defined via transpositions and does not create a cycle even for partitions of length 3, because different permutations show up with different coefficients. Our definition of Perm seems to be the intended one.

Let $\left[n_{1}, \ldots, n_{s}\right]$ be any partition of $n$. Then the alternating sum of its permutations

$$
\sum_{\sigma \in S_{s}} \operatorname{sign}(\sigma)\left[n_{\sigma(1)}, \ldots, n_{\sigma(s)}\right]
$$

is a cycle in $A_{n}^{\bullet}$. With $\mathbb{Z}_{p}$-coefficients, the following subset of permutations

$$
\operatorname{Perm}\left[n_{1}, \ldots, n_{s}\right]=\sum_{\begin{array}{l}
\sigma \in S_{s} \text { where } \sigma(i)<\sigma(j) \\
\text { if } i<j \text { and } n_{i}=n_{j} \text { or } \\
\text { if } i<j \text { and } P\left(n_{i}, n_{j}\right)=0 \bmod p
\end{array}} \operatorname{sign}(\sigma)\left[n_{\sigma(1)}, \ldots, n_{\sigma(s)}\right]
$$

will be used in the next paragraph to create special cycles in $A_{n}^{\bullet} \otimes \mathbb{Z}_{p}$.

Take integers $1 \leqslant i_{1} \leqslant \cdots \leqslant i_{k}$ and $0 \leqslant j_{1}<\cdots<j_{l}$ such that

$$
t=n-2\left(p^{i_{1}}+\cdots+p^{i_{k}}+p^{j_{1}}+\cdots+p^{j_{l}}\right) \geqslant 0
$$

and let

$$
r=\left(2 p^{i_{1}}-2\right)+\cdots+\left(2 p^{i_{k}}-2\right)+\left(2 p^{j_{1}}-1\right)+\cdots+\left(2 p^{j_{l}}-1\right) .
$$

Then we give the chain

$$
\operatorname{Ins}_{t} \operatorname{Perm}\left[2 p^{i_{1}-1}, 2 p^{i_{1}-1}(p-1), \ldots, 2 p^{i_{k}-1}, 2 p^{i_{k}-1}(p-1), 2 p^{j_{1}}, \ldots, 2 p^{j_{l}}\right]
$$

the name $x_{i_{1}} \cdots x_{i_{k}} y_{j_{1}} \cdots y_{j_{l}}$. It is a cycle in $A_{n, t}^{r} \otimes \mathbb{Z}_{p}$ (but not in $A_{n}^{\bullet}$ if $k>0$ ). Vainshtein showed that all such cycles form a basis of $H^{r}\left(A_{n}^{\bullet}, \mathbb{Z}_{p}\right)$. We call the quantity $n-t$ the size of the chain $x_{i_{1}} \cdots x_{i_{k}} y_{j_{1}} \cdots y_{j_{l}}$.

Theorem $2.3([25])$. The group $H^{*}\left(C_{\infty}, \mathbb{Z}_{p}\right)$ is the free graded commutative algebra over $\mathbb{Z}_{p}$ with generators

$$
\begin{array}{lll}
x_{i} \text { for } i \geqslant 1 & \operatorname{deg}\left(x_{i}\right)=2 p^{i}-2 & \operatorname{size}\left(x_{i}\right)=2 p^{i}, \\
y_{i} \text { for } i \geqslant 0 & \operatorname{deg}\left(y_{i}\right)=2 p^{i}-1 & \operatorname{size}\left(y_{i}\right)=2 p^{i} .
\end{array}
$$

There is a surjection $H^{*}\left(C_{\infty}(\mathbb{C}), \mathbb{Z}_{p}\right) \rightarrow H^{*}\left(C_{n}(\mathbb{C}), \mathbb{Z}_{p}\right)$ whose kernel is generated by the monomials $x_{i_{1}} \cdots x_{i_{k}} y_{j_{1}} \cdots y_{j_{l}}$ such that $\operatorname{size}\left(x_{i_{1}} \cdots x_{i_{k}} y_{j_{1}} \cdots y_{j_{l}}\right)>n$.

A equivalent formula was deduced by Cohen-Lada-May [8, Appendix to III].

Remark 2.4. For $p=2$, the group $H^{*}\left(C_{\infty}, \mathbb{Z}_{2}\right)$ can be identified with a polynomial algebra with generators

$$
z_{i} \text { for } i \geqslant 1, \quad \operatorname{deg}\left(z_{i}\right)=2^{i}-1
$$

via $x_{i} \mapsto z_{i}^{2}$ and $y_{i} \mapsto z_{i-1}$. This is the form stated in [8]. 
Corollary 2.5. Define

$$
B_{p}(n, r)=\left|\left\{\begin{array}{c|c}
1 \leqslant a_{1} \leqslant a_{2} \leqslant \cdots \leqslant a_{g} & 2 \sum_{i} p^{a_{i}}+2 \sum_{j} p^{b_{j}}-2 g-h=r \\
0 \leqslant b_{1}<b_{2}<\cdots<b_{h} & 2 \sum_{i} p^{a_{i}}+2 \sum_{j} p^{b_{j}} \leqslant n
\end{array}\right\}\right| .
$$

Hence we have

$$
\operatorname{dim} H^{r}\left(C_{n}(\mathbb{C}), \mathbb{Z}_{p}\right)=B_{p}(n, r) .
$$

Remark 2.6. Paolo Salvatore (private communication) gave this representation as a generating series:

$$
\sum_{n, r \geqslant 0} B_{p}(n, r) w^{r} z^{n}=\frac{1+w z^{2}}{1-z} \prod_{i>0} \frac{1+w^{2 p^{i}-1} z^{2 p^{i}}}{1-w^{2 p^{i}-2} z^{2 p^{i}}} .
$$

Remark 2.7. The notation suggests a product structure on $H^{*}\left(C_{\infty}(\mathbb{C}), \mathbb{Z}_{p}\right)$. It comes from the map

$$
C_{n}(\mathbb{C}) \times C_{m}(\mathbb{C}) \rightarrow C_{n+m}(\mathbb{C})
$$

by adding the points far apart. However, in this paper we will use it only as a convenient notation.

Remark 2.8. As

$$
\left(\begin{array}{c}
p^{a}+p^{b} \\
p^{a}
\end{array}\right) \equiv\left\{\begin{array}{ll}
1 & a \neq b \\
2 & a=b
\end{array} \quad \bmod p\right.
$$

and

$$
\left(\begin{array}{c}
p^{a}+p^{b}(p-1) \\
p^{a}
\end{array}\right) \equiv\left\{\begin{array}{ll}
1 & a \neq b \\
0 & a=b
\end{array} \quad \bmod p\right.
$$

by Lucas's Theorem [12], the order of all entries of the form $2 p^{a}, 2 p^{a}(p-1)$ in our basis elements is preserved by the operator Perm. All other entries are permuted.

Example 2.9. In order to give an example for all the constructions, we compute $H^{*}\left(C_{24}(\mathbb{C}), \mathbb{Z} / 3 \mathbb{Z}\right)$. The generators have degrees:

\begin{tabular}{ccccccc} 
generators & $x_{1}$ & $x_{2}$ & $y_{0}$ & $y_{1}$ & $y_{2}$ & $\ldots$ \\
\hline degree & 4 & 16 & 1 & 5 & 17 & $\ldots$ \\
size & 6 & 18 & 2 & 6 & 18 & $\ldots$
\end{tabular}

In Table 1, we write down the basis elements and the corresponding chains, however, we will omit the application of the $\mathrm{Ins}_{t}$-operators to lift the chains to sum 24 .

\subsection{Bockstein homomorphisms}

The short exact sequences of coefficients

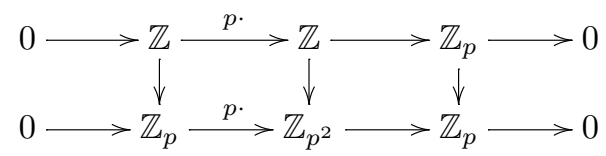


Table 1: The cohomology group $H^{*}\left(C_{24}(\mathbb{C}), \mathbb{Z}_{3}\right)$

\begin{tabular}{|c|c|}
\hline 0 & $1=[]$ \\
\hline 1 & $y_{0}=[2]$ \\
\hline 2 & - \\
\hline 3 & - \\
\hline 4 & $x_{1}=[2,4]$ \\
\hline 5 & $\begin{array}{l}y_{1}=[6] \\
x_{1} y_{0}=[2,4,2]\end{array}$ \\
\hline 6 & $y_{0} y_{1}=[2,6]-[6,2]$ \\
\hline 7 & - \\
\hline 8 & $x_{1}^{2}=[2,4,2,4]$ \\
\hline 9 & $\begin{array}{l}x_{1} y_{1}=[2,4,6]-[2,6,4]+[6,2,4] \\
x_{1}^{2} y_{0}=[2,4,2,4,2]\end{array}$ \\
\hline 10 & $x_{1} y_{0} y_{1}=[2,4,2,6]-[2,4,6,2]+[2,6,4,2]-[6,2,4,2]$ \\
\hline 11 & - \\
\hline 12 & $x_{1}^{3}=[2,4,2,4,2,4]$ \\
\hline 13 & $\begin{array}{l}x_{1}^{2} y_{1}=[2,4,2,4,6]-[2,4,2,6,4]+[2,4,6,2,4]-[2,6,4,2,4]+[6,2,4,2,4] \\
x_{1}^{3} y_{0}=[2,4,2,4,2,4,2]\end{array}$ \\
\hline 14 & $x_{1}^{2} y_{0} y_{1}=[2,4,2,4,2,6]-[2,4,2,4,6,2]+[2,4,2,6,4,2]-[2,4,6,2,4,2]+,\cdots$ \\
\hline 15 & - \\
\hline 16 & $\begin{array}{l}x_{2}=[6,12] \\
x_{1}^{4}=[2,4,2,4,2,4,2,4]\end{array}$ \\
\hline 17 & $\begin{array}{l}y_{2}=[18] \\
x_{2} y_{0}=[6,12,2]-[6,2,12]+[2,6,12] \\
x_{1}^{3} y_{1}=[2,4,2,4,2,4,6]-[2,4,2,4,2,6,4]+\cdots\end{array}$ \\
\hline 18 & $y_{0} y_{2}=[2,18]-[18,2]$ \\
\hline 19 & - \\
\hline 20 & $x_{1} x_{2}=[2,4,6,12]-[2,6,4,12]+[6,2,4,12]-[6,2,12,4]+[2,6,12,4]+[6,12,2,4]$ \\
\hline 21 & $\begin{array}{l}x_{1} y_{2}=[2,4,18]-[2,18,4]+[18,2,4] \\
x_{2} y_{1}=[6,12,6]\end{array}$ \\
\hline 22 & $y_{1} y_{2}=[6,18]-[18,6]$ \\
\hline 23 & - \\
\hline
\end{tabular}

induce long exact sequences

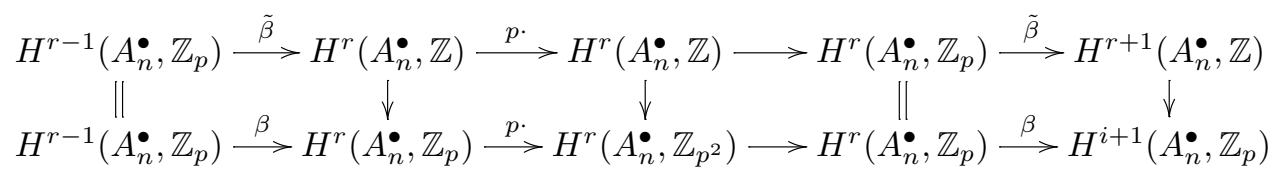

where the connecting morphisms are the Bockstein morphisms $\beta$ and $\tilde{\beta}$ (compare [15, Chap. 3.E]). The image of $\tilde{\beta}$ hence consists of all the elements of order $p$ in $H^{*}\left(A_{n}^{\bullet}, \mathbb{Z}\right)$. 
Example 2.10. Let $i \neq j$. We determine the Bockstein on

$$
x_{i}=\left[2 p^{i-1}, 2 p^{i-1}(p-1)\right]
$$

and

$x_{i} y_{j}=\left[2 p^{i-1}, 2 p^{i-1}(p-1), 2 p^{j}\right]-\left[2 p^{i-1}, 2 p^{j}, 2 p^{i-1}(p-1)\right]+\left[2 p^{j}, 2 p^{i-1}, 2 p^{i-1}(p-1)\right]$.

In $A_{n}^{\bullet}$, we get

$$
\begin{aligned}
\delta\left(x_{i}\right) & =\left(\begin{array}{c}
p^{i} \\
p^{i-1}
\end{array}\right)\left[2 p^{i}\right]=\left(\begin{array}{c}
p^{i} \\
p^{i-1}
\end{array}\right) y_{i} \\
\delta\left(x_{i} y_{j}\right) & =\left(\begin{array}{c}
p^{i} \\
p^{i-1}
\end{array}\right)\left(\left[2 p^{i}, 2 p^{j}\right]-\left[2 p^{j}, 2 p^{i}\right]\right)=\left(\begin{array}{c}
p^{i} \\
p^{i-1}
\end{array}\right) y_{i} y_{j} .
\end{aligned}
$$

Hence we can conclude

$$
\tilde{\beta}\left(x_{i}\right)=\frac{1}{p}\left(\begin{array}{c}
p^{i} \\
p^{i-1}
\end{array}\right) y_{i}, \quad \tilde{\beta}\left(x_{i} y_{j}\right)=\frac{1}{p}\left(\begin{array}{c}
p^{i} \\
p^{i-1}
\end{array}\right) y_{i} y_{j} .
$$

The coefficient

$$
\frac{1}{p}\left(\begin{array}{c}
p^{i} \\
p^{i-1}
\end{array}\right)=\left(\begin{array}{c}
p^{i}-1 \\
p^{i-1}-1
\end{array}\right)
$$

is an integer congruent to $1 \bmod p$ by Lucas' Theorem [12].

Lemma 2.11. The differential $\delta$ on $A_{n}^{\bullet}$ operates as follows:

$$
\delta\left(x_{1}^{a_{1}} \cdots x_{k}^{a_{k}} y_{0}^{b_{1}} \cdots y_{l}^{b_{l}}\right)=\sum_{i}\left(\begin{array}{c}
p^{i} \\
p^{i-1}
\end{array}\right) x_{1}^{a_{1}} \cdots x_{i}^{a_{i}-1} \cdots x_{k}^{a_{k}} y_{i} y_{0}^{b_{0}} \cdots y_{l}^{b_{l}} .
$$

Hence the Bocksteins are given by

$$
\tilde{\beta}\left(x_{1}^{a_{1}} \cdots x_{k}^{a_{k}} y_{0}^{b_{1}} \cdots y_{l}^{b_{l}}\right)=\frac{1}{p} \sum_{i}\left(\begin{array}{c}
p^{i} \\
p^{i-1}
\end{array}\right) x_{1}^{a_{1}} \cdots x_{i}^{a_{i}-1} \cdots x_{k}^{a_{k}} y_{i} y_{0}^{b_{0}} \cdots y_{l}^{b_{l}}
$$

and

$$
\beta\left(x_{1}^{a_{1}} \cdots x_{k}^{a_{k}} y_{0}^{b_{1}} \cdots y_{l}^{b_{l}}\right)=\sum_{i} x_{1}^{a_{1}} \cdots x_{i}^{a_{i}-1} \cdots x_{k}^{a_{k}} y_{i} y_{0}^{b_{0}} \cdots y_{l}^{b_{l}} .
$$

Proof. Let $m=x_{1}^{a_{1}} \cdots x_{k}^{a_{k}} y_{0}^{b_{1}} \cdots y_{l}^{b_{l}}$. Take any term $\left[\ldots, n_{1}, n_{2}, \ldots\right]$ in $m$. It only contributes $\left[\ldots, n_{1}+n_{2}, \ldots\right]$ to $\delta(m)$ if $\left[n_{1}, n_{2}\right]=\left[2 p^{i-1}, 2(p-1) p^{i-1}\right]$ or $\left[n_{1}, n_{2}\right]=$ $\left[2(p-1) p^{i-1}, p^{i-1}\right]$. Otherwise, $\left[\ldots, n_{1}+n_{2}, \ldots\right]$ is cancelled by $\delta\left(\left[\ldots, n_{2}, n_{1}, \ldots\right]\right)$ as $\left[\ldots, n_{2}, n_{1}, \ldots\right]$ shows up in $m$ with opposite sign due to the definition of Perm. Now

$$
\delta\left(\left[\ldots, 2 p^{i-1}, 2(p-1) p^{i-1}, \ldots\right]\right)= \pm\left(\begin{array}{c}
p^{i} \\
p^{i-1}
\end{array}\right)\left[\ldots, 2 p^{i}, \ldots\right]+\cdots,
$$

and a tedious calculation of signs proves the formula.

As $\beta^{2}=0$, we can look at the Bockstein cohomology groups

$$
B H^{*}\left(A_{n}^{\bullet}, \mathbb{Z}_{p}\right)=\operatorname{Ker} \beta / \operatorname{Im} \beta .
$$

Lemma 2.12 ([15, Cor. 3E.4]). The group $H^{*}\left(A_{n}^{\bullet}, \mathbb{Z}\right)$ contains no element of order $p^{2}$ if and only if

$$
\operatorname{dim}_{\mathbb{Z}_{p}} B H^{r}\left(A_{n}^{\bullet}, \mathbb{Z}_{p}\right)=\operatorname{rk} H^{r}\left(A_{n}^{\bullet}, \mathbb{Z}\right)
$$


In this case the map

$$
H^{*}\left(A_{n}^{\bullet}, \mathbb{Z}\right) \rightarrow H^{*}\left(A_{n}^{\bullet}, \mathbb{Z}_{p}\right)
$$

is injective on the p-torsion and its image is $\operatorname{Im} \beta$.

Vainshtein stated that $H^{*}\left(A_{n}^{\bullet}, \mathbb{Z}\right)$ has no elements of order $p^{2}$ :

Theorem 2.13 ([25]). The integral cohomology is given by

$$
H^{0}\left(C_{n}(\mathbb{C}), \mathbb{Z}\right)=\mathbb{Z}, \quad H^{1}\left(C_{n}(\mathbb{C}), \mathbb{Z}\right)=\mathbb{Z} \text { if } n \geqslant 2
$$

and

$$
H^{r}\left(C_{n}(\mathbb{C}), \mathbb{Z}\right)=\bigoplus_{p} \tilde{\beta}_{p} H^{r-1}\left(C_{n}(\mathbb{C}), \mathbb{Z}_{p}\right) \text { for } r \geqslant 2 .
$$

Proof. Take any $x \in \operatorname{Ker} \beta$ of the form

$$
x=x_{j}^{k} f+x_{j}^{k-1} y_{j} g
$$

for $k \geqslant 0, j>0$ where $f, g$ do not contain $x_{j}$ or $y_{j}$. We compute

$$
\beta(x)=x_{j}^{k-1} y_{j} f+x_{j}^{k} \beta(f)-x_{j}^{k-1} y_{j} \beta(g) .
$$

Hence we see $\beta(g)=f$ and $\beta\left(x_{j}^{k} g\right)=x$. So we have shown that

$$
\operatorname{Ker} \beta / \operatorname{Im} \beta=\mathbb{Z}_{p} \oplus \mathbb{Z}_{p} y_{0} \text {. }
$$

Remark 2.14. The map $\beta$ arises as the reduction $\bmod p$ of the unique graded derivation $\beta^{\prime}$ on the free divided power algebra $[15$, Ex 3.5C] on generators

$$
x_{1}, x_{2}, \ldots, y_{0}, y_{1}, \ldots \quad \operatorname{deg}\left(x_{i}\right)=2 p^{i}-2 \quad \operatorname{deg}\left(y_{i}\right)=2 p^{i}-1,
$$

with multiplication $x_{i}^{j_{1}} \star x_{i}^{j_{2}}=\left(\begin{array}{c}j_{1}+j_{2} \\ j_{1}\end{array}\right) x_{i}^{j_{1}+j_{2}}$ where $\beta^{\prime}$ is given by the formulas

$$
\beta^{\prime}\left(X_{i}\right)=Y_{i}, \quad \beta^{\prime}\left(Y_{i}\right)=0
$$

and the rule (compare [11, Chap. 3])

$$
\beta^{\prime}\left(z_{1} \star z_{2}\right)=\beta^{\prime}\left(z_{1}\right) \star z_{2}+(-1)^{\operatorname{deg} z_{1}} z_{1} \star \beta^{\prime}\left(z_{2}\right) .
$$

Corollary 2.15. The $p$-Torsion of $H^{r+1}\left(C_{\infty}(\mathbb{C}), \mathbb{Z}\right)$ is isomorphic to the degree $r$ part of the free graded algebra over $\mathbb{Z}_{p}$ with generators $x_{1}, x_{2}, \ldots, y_{1}, y_{2}, \ldots>$ for $r>0$.

Proof. Write $R$ for the free graded algebra over $\mathbb{Z}_{p}$ with generators $x_{1}, x_{2}, \ldots, y_{1}$, $y_{2}, \ldots$ Theorem 2.3 shows that

$$
H^{*}\left(C_{\infty}(\mathbb{C}), \mathbb{Z}_{p}\right)=R \oplus y_{0} R .
$$

By Lemma 2.11 we know that $\beta\left(x y_{0}\right)=\beta(x) y_{0}$ and $\beta(R) \subset R$. This shows

$$
\operatorname{Im} \beta=\beta(R) \oplus y_{0} \beta(R) .
$$

Decompose $R=\beta(R) \oplus R^{\prime}$. As $\operatorname{Ker} \beta=\operatorname{Im} \beta \oplus \mathbb{Z}_{p} \oplus \mathbb{Z}_{p} y_{0}$, the map

$$
\beta(R) \oplus R^{\prime} \rightarrow \beta(R) \oplus y_{0} \beta(R)=\operatorname{Im} \beta,\left(z_{1}, z_{2}\right) \mapsto \beta\left(z_{2}\right)+y_{0} z_{1}
$$

is a bijective map between the degree $r$ part of $R$ and the degree $r+1$ part of $\operatorname{Im} \beta$ for $r>0$.

However, it does not respect the size, so this isomorphism allows to describe the p-Torsion of $H^{r+1}\left(C_{\infty}(\mathbb{C}), \mathbb{Z}\right)$, but not of $H^{r+1}\left(C_{n}(\mathbb{C}), \mathbb{Z}\right)$ for $n<\infty$. 
Remark 2.16. The description of the dimension of the $p$-torsion of $H^{r}\left(C_{n}(\mathbb{C}), \mathbb{Z}\right)$ in [8, Appendix to III, Cor. A4] seems to be wrong. For example, we can compute that $H^{21}\left(C_{\infty}(\mathbb{C}), \mathbb{Z}_{3}\right)$ would be 5 -dimensional, the 3 -Torsion in $H^{20}\left(C_{\infty}(\mathbb{C}), \mathbb{Z}\right)$ would be 2-dimensional and the 3 -Torsion in $H^{21}\left(C_{\infty}(\mathbb{C}), \mathbb{Z}_{3}\right)$ would also be 2-dimensional. This contradicts the universal coefficient theorem.

The description of $H^{*}\left(C_{n}(\mathbb{C}), \mathbb{Z}_{p}\right)$ and of the Bockstein homomorphism in [8, Appendix to III] is correct, however, the image of the Bockstein is not given by the subalgebra described there in Corollary A4. A simple formula for the dimension of $H^{r}\left(C_{n}(\mathbb{C}), \mathbb{Z}\right)$ probably does not exist.

Example 2.17. In Table 2, we compute $H^{*}\left(C_{24}(\mathbb{C}), \mathbb{Z}_{3}\right)_{T_{3}}$ by applying Theorem 2.13 and Formula 2.11 to our Example 2.9.

Table 2: The 3-torsion in the cohomology group $H^{*}\left(C_{24}(\mathbb{C}), \mathbb{Z}\right)$

\begin{tabular}{l|l}
$r$ & basis of $H^{r}\left(C_{24}(\mathbb{C}), \mathbb{Z}\right)_{T_{3}}$ as $\mathbb{Z}_{3}$-module \\
\hline \hline 0 & - \\
\hline 1 & - \\
\hline 2 & - \\
\hline 3 & - \\
\hline 4 & - \\
\hline 5 & $y_{1}=[6]$ \\
\hline 6 & $y_{0} y_{1}=[2,6]-[6,2]$ \\
\hline 7 & - \\
\hline 8 & \\
\hline 9 & $x_{1} y_{1}=[2,4,6]-[2,6,4]+[6,2,4]$ \\
\hline 10 & $x_{1} y_{0} y_{1}=[2,4,2,6]-[2,4,6,2]+[2,6,4,2]-[6,2,4,2]$ \\
\hline 11 & - \\
\hline 12 & - \\
\hline 13 & $x_{1}^{2} y_{1}=[2,4,2,4,6]-[2,4,2,6,4]+[2,4,6,2,4]-[2,6,4,2,4]+[6,2,4,2,4]$ \\
\hline 14 & $x_{1}^{2} y_{0} y_{1}=[2,4,2,4,2,6]-[2,4,2,4,6,2]+[2,4,2,6,4,2]-[2,4,6,2,4,2]+,\cdots$ \\
\hline 15 & - \\
\hline 16 & - \\
\hline 17 & $y_{2}=[18]$ \\
\hline 18 & $x_{1}^{3} y_{1}=[2,4,2,4,2,4,6]-\cdots$ \\
\hline 19 & $y_{0} y_{2}=[2,18]-[18,2]$ \\
\hline 20 & - \\
\hline 21 & $28 x_{1} y_{2}+x_{2} y_{1}=28([2,4,18]-[2,18,4]+[18,2,4])+[6,12,6]$ \\
\hline 22 & $y_{1} y_{2}=[6,18]-[18,6]$ \\
\hline$\geqslant 23$ & - \\
& \\
\hline 12
\end{tabular}

\section{Configuration spaces of the sphere}

We will describe a cellular decomposition of $C_{n}\left(S^{2}\right)^{+}$by Napolitano [18] and show how it can be used to compute the cohomology of $C_{n}\left(S^{2}\right)$. 


\subsection{Cellular decomposition of $C_{n}\left(S^{2}\right)^{+}$}

The cellular decomposition of $C_{n}(\mathbb{C})^{+}$can be extended to a cellular decomposition of $C_{n}\left(S^{2}\right)^{+}$. Using $S^{2}=\mathbb{R}^{2} \cup \infty$, we see that $n$ points on $S^{2}$ are either $n$ points on $\mathbb{R}^{2}$ or $n-1$ points on $\mathbb{R}^{2}$ and the point $\infty$. So the cells of $C_{n}\left(S^{2}\right)$ are the union of the cells of $C_{n}\left(\mathbb{R}^{2}\right)$ and $C_{n-1}\left(\mathbb{R}^{2}\right)$. The resulting complex $B_{n}^{\bullet}=\left(B_{n}^{r}\right)$ with $H^{*}\left(B_{n}^{\bullet}, \mathbb{Z}\right)=$ $H^{*}\left(C_{n}\left(S^{2}\right), \mathbb{Z}\right)$ has chains

$$
B_{n}^{r}=A_{n}^{r} \oplus A_{n-1}^{r-2}=\mathbb{Z} \operatorname{Part}(n, n-r) \oplus \mathbb{Z} \operatorname{Part}(n-1, n-r+1) .
$$

The new boundary maps $\Delta$ were computed by Napolitano [18]. We define a new operator $D: A_{n}^{r} \rightarrow A_{n-1}^{r-1}$ by

$$
D\left[n_{1}, \ldots, n_{s}\right]=\sum_{i=1}^{s} Q\left(n_{i}\right)(-1)^{\sum_{j=1}^{i-1} n_{j}}\left[n_{1}, \ldots, n_{i-1}, n_{i}-1, n_{i+1}, \ldots, n_{s}\right],
$$

where

$$
Q\left(n_{i}\right)= \begin{cases}0 & \text { if } n_{i} \equiv 1 \quad \bmod 2, \\ 2 & \text { otherwise }\end{cases}
$$

The differential $\Delta$ of the complex $B_{n}^{\bullet}$ is then given by

$$
\Delta: B_{n}^{r} \rightarrow B_{n}^{r+1},(a, b) \mapsto\left(\delta(a), \delta(b)+(-1)^{n-r} D(a)\right) .
$$

Corollary 3.1. We have $D \equiv 0 \bmod 2$ and therefore $B_{n}^{\bullet} \otimes \mathbb{Z}_{2}=\left(A_{n}^{\bullet} \oplus A_{n-1}^{\bullet}\right) \otimes \mathbb{Z}_{2}$ and

$$
H^{r}\left(C_{n}\left(S^{2}\right), \mathbb{Z}_{2}\right)=H^{r}\left(C_{n}(\mathbb{C}), \mathbb{Z}_{2}\right) \oplus H^{r-2}\left(C_{n-1}(\mathbb{C}), \mathbb{Z}_{2}\right) .
$$

The groups $H^{r}\left(C_{n}\left(S^{2}\right), \mathbb{Z}_{2}\right)$ have already been determined in [4].

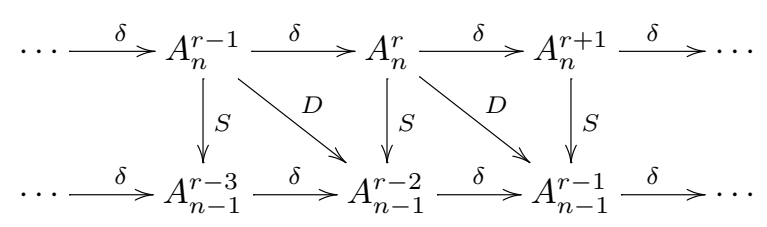

\subsection{Mapping cone complex}

Lemma 3.2. We get a long exact sequence

$$
\cdots \rightarrow H^{r-1}\left(A_{n}^{\bullet} \stackrel{D^{*}}{\longrightarrow} H^{r-2}\left(A_{n-1}^{\bullet}\right) \rightarrow H^{r}\left(B_{n}^{\bullet}\right) \rightarrow H^{r}\left(A_{n}^{\bullet}\right) \stackrel{D^{*}}{\longrightarrow} H^{r-1}\left(A_{n-1}^{\bullet}\right) \rightarrow \cdots .\right.
$$

Proof. The relation

$$
D \circ \delta=\delta \circ D
$$

is equivalent to $\Delta^{2}=0$. This means we can see $D$ as a chain map

$$
D: A_{n}^{\bullet} \rightarrow A_{n-1}^{\bullet}[1]
$$

and the complex $B_{n}^{\bullet}$ can be interpreted as the mapping cone complex of the chain map $D$. The short exact sequence of chain complexes

$$
0 \rightarrow A_{n-1}^{\bullet}[2] \rightarrow B_{n}^{\bullet} \rightarrow A_{n}^{\bullet} \rightarrow 0
$$

given by $a_{2} \mapsto\left(0, a_{2}\right)$ and $\left(a_{1}, a_{2}\right) \mapsto a_{1}$ induces a long exact sequence with a connecting homomorphism that can be identified with $D^{*}$. 
We can use this long exact sequence to compare the cohomology of $B_{n}^{\bullet}, A_{n}^{\bullet}$ and $A_{n-1}^{\bullet}$. Next we will construct a map

$$
S: A_{n}^{r} \rightarrow A_{n-1}^{r-2},
$$

which is almost a chain homotopy $D \approx 2 \delta S+2 S \delta$ between $D$ and the zero map. This allows us to compute the rank of $D^{*}$.

\section{Construction of (almost) a null homotopy}

As a motivation we first look at the case $r=n-1$. We set $S[n]=[1, n-2]$. Then we have

$$
2 \delta S[n]=2 \delta[1, n-2]=2[n-1]=D[n]
$$

if $n$ is even and

$$
2 \delta S[n]=2 \delta[1, n-2]=0=D[n]
$$

otherwise.

In general, we define $S: A_{n}^{r} \rightarrow A_{n-1}^{r-2}$ by

$S\left[n_{1}, \ldots, n_{s}\right]=\sum_{1 \leqslant k \leqslant i \leqslant s}(-1)^{k+1+\sum_{m=1}^{k-1} n_{m}}\left[n_{1}, \ldots, n_{k-1}, 1, n_{k}, \ldots, n_{i-1}, n_{i}-2, n_{i+1}, \ldots, n_{s}\right]$.

If $n_{i}-2 \leqslant 0$, we simply omit this summand. We remark, that all calculations in this chapter are done on the chain level.

Lemma 4.1. Define $E=D-2 \delta \circ S-2 S \circ \delta$. For every partition $\left[n_{1}, \ldots, n_{s}\right]$ with $n_{s} \neq 2$ we have

$$
E\left[n_{1}, \ldots, n_{s}\right]=0
$$

and

$$
E\left[n_{1}, \ldots, n_{s-1}, 2\right]=2 \sum_{1 \leqslant k \leqslant s}(-1)^{s+k+\sum_{m=1}^{k-1} n_{m}}\left[n_{1}, \ldots, n_{k-1}, 1, n_{k}, \ldots, n_{s-1}\right]
$$

otherwise.

Proof. For convenience we introduce the operators $\delta_{l}$ by

$$
\delta_{l}\left[m_{1}, \ldots, m_{t}\right]=(-1)^{l-1} P\left(m_{l}, m_{l+1}\right)\left[m_{1}, \ldots, m_{l-1}, m_{l}+m_{l+1}, m_{l+2}, \ldots, m_{t}\right]
$$

and the abbreviations

$$
n_{k, i}=(-1)^{k+1+\sum_{m=1}^{k-1} n_{m}}\left[n_{1}, \ldots, n_{k-1}, 1, n_{k}, \ldots, n_{i-1}, n_{i}-2, n_{i+1}, \ldots, n_{s}\right] .
$$

Let us first assume that all $n_{i}>2$. We compute

$$
\delta \circ S\left[n_{1}, \ldots n_{r}\right]=\sum_{\substack{1 \leqslant l \leqslant s \\ k \leqslant i}} \delta_{l}\left(n_{k, i}\right)
$$

by splitting up the index set

$$
I=\{1 \leqslant l \leqslant s, 1 \leqslant k \leqslant i \leqslant s\}
$$

into

$$
I=I_{1} \sqcup \cdots \sqcup I_{8},
$$


where

$$
\begin{array}{ll}
I_{1}=\{1 \leqslant l<k-1, k \leqslant i\}, & I_{5}=\{l=i+1, k \leqslant i\}, \\
I_{2}=\{k+1 \leqslant l<i\}, & I_{6}=\{l=k-1, k \leqslant i\}, \\
I_{3}=\{i+2 \leqslant l \leqslant s, k \leqslant i\}, & I_{7}=\{l=k, k<i\}, \\
I_{4}=\{l=i, k<i\}, & I_{8}=\{l=k=i\} .
\end{array}
$$

Now we look at the individual summands $T_{j}=\sum_{I_{j}} \delta_{l}\left(n_{k, i}\right)$ and expand them after doing some index shifts. Write ind $=k+l+\sum_{m=1}^{k-1} n_{m}$.

$$
\begin{aligned}
& T_{1}=\sum_{\substack{l<k-1 \\
k \leqslant i}}(-1)^{\mathrm{ind}} P\left(n_{l}, n_{l+1}\right)\left[\ldots, n_{l}+n_{l+1}, \ldots, n_{k-1}, 1, n_{k}, \ldots, n_{i-1}, n_{i}-2, n_{i+1}, \ldots\right] \\
& T_{2}=\sum_{k \leqslant l<i-1}(-1)^{\mathrm{ind}+1} P\left(n_{l}, n_{l+1}\right)\left[\ldots, n_{k-1}, 1, n_{k}, \ldots, n_{l}+n_{l+1}, \ldots, n_{i-1}, n_{i}-2, n_{i+1}, \ldots\right] \\
& T_{3}=\sum_{k \leqslant i<l}(-1)^{\text {ind }+1} P\left(n_{l}, n_{l+1}\right)\left[\ldots, n_{k-1}, 1, n_{k}, \ldots, n_{i-1}, n_{i}-2, n_{i+1}, \ldots, n_{l}+n_{l+1}, \ldots\right]
\end{aligned}
$$

The next terms

$$
\begin{aligned}
T_{4} & =\sum_{k<i}(-1)^{k+i+\sum_{m=1}^{k-1} n_{m}} P\left(n_{i-1}, n_{i}-2\right)\left[\ldots, n_{k-1}, 1, n_{k}, \ldots, n_{i-1}+n_{i}-2, n_{i+1}, \ldots\right] \\
T_{5} & =\sum_{k \leqslant i}(-1)^{k+i+1+\sum_{m=1}^{k-1} n_{m}} P\left(n_{i}-2, n_{i+1}\right)\left[\ldots, n_{k-1}, 1, n_{k}, \ldots, n_{i-1}, n_{i}-2+n_{i+1}, \ldots\right]
\end{aligned}
$$

sum up to

$T_{4}+T_{5}=\sum_{k \leqslant i}(-1)^{k+i+1+\sum_{m=1}^{k-1} n_{m}} P\left(n_{i}, n_{i+1}\right)\left[\ldots, n_{k-1}, 1, n_{k}, \ldots, \ldots, n_{i-1}, n_{i}-2+n_{i+1}, \ldots\right]$

where we use the identity $P(x-2, y)+P(x, y-2)=P(x, y)$. Altogether we have

$$
T_{1}+T_{2}+T_{3}+T_{4}+T_{5}=-S \circ \delta\left[n_{1}, \ldots, n_{s}\right] .
$$

The terms

$$
\begin{aligned}
& T_{6}=\sum_{k \leqslant i}(-1)^{2 k-2+\sum_{m=1}^{k-1} n_{m}} P\left(n_{k-1}, 1\right)\left[\ldots, n_{k-2}, n_{k-1}+1, n_{k}, \ldots, n_{i-1}, n_{i}-2, n_{i+1}, \ldots\right], \\
& T_{7}=\sum_{k<i}(-1)^{2 k-1+\sum_{m=1}^{k-1} n_{m}} P\left(1, n_{k}\right)\left[\ldots, n_{k-1}, 1+n_{k}, n_{k+1}, \ldots, n_{i-1}, n_{i}-2, n_{i+1}, \ldots\right]
\end{aligned}
$$

contain the same summands with alternating signs and cancel each other. For the remaining summand

$$
T_{8}=\sum_{i}(-1)^{\sum_{m=1}^{i-1} n_{m}} P\left(1, n_{i}-2\right)\left[\ldots, n_{i-1}, n_{i}-1, n_{i+1}, \ldots\right],
$$

the following equation holds

$$
2 T_{8}=D\left[n_{1}, \ldots, n_{s}\right]
$$

by the definition of $D$. Here we use $P\left(1, n_{i}-2\right)=1$ if $n_{i}$ even and $P\left(1, n_{i}-2\right)=0$ if $n_{i}$ odd. In the end we get

$$
2 \delta \circ S\left[n_{1}, \ldots, n_{s}\right]=-2 S \circ \delta\left[n_{1}, \ldots, n_{s}\right]+D\left[n_{1}, \ldots, n_{s}\right] .
$$

In case that $n_{j}=2$ with $j<s$, all contributions containing $n_{j}-2$ in $T_{4}, T_{5}$ and 
$T_{8}$ are missing in $\delta \circ S$, but not in $S \circ \delta$ and $D$. So we have to add

$$
\begin{aligned}
T_{4}^{\prime} & =\sum_{k<j}(-1)^{k+j+\sum_{m=1}^{k-1} n_{m}} P\left(n_{j-1}, 0\right)\left[\ldots, 1, n_{k}, \ldots, n_{j-2}, n_{j-1}, n_{j+1}, \ldots\right], \\
T_{5}^{\prime} & =\sum_{k \leqslant j}(-1)^{k+j+1+\sum_{m=1}^{k-1} n_{m}} P\left(0, n_{j+1}\right)\left[\ldots, 1, n_{k}, \ldots, n_{j-1}, n_{j+1}, \ldots\right], \\
T_{8}^{\prime} & =(-1)^{\sum_{m=1}^{j-1} n_{m}} P(1,0)\left[\ldots, n_{j-1}, 1, n_{j+1}, \ldots\right],
\end{aligned}
$$

which simplifies using $P(x, 0)=1$ and $P(0, y)=1$ to:

$$
\begin{aligned}
T_{4}^{\prime}+T_{8}^{\prime} & =\sum_{k \leqslant j}(-1)^{k+j+\sum_{m=1}^{k-1} n_{m}}\left[\ldots, n_{k-1}, 1, n_{k}, n_{j-2}, \ldots, n_{j-1}, n_{j+1}, \ldots\right], \\
T_{5}^{\prime} & =\sum_{k \leqslant j}(-1)^{k+j+1+\sum_{m=1}^{k-1} n_{m}}\left[\ldots, n_{k-1}, 1, n_{k}, \ldots, n_{j-1}, n_{j+1}, \ldots\right] .
\end{aligned}
$$

Hence we have

$$
(D-2 \delta \circ S-2 S \circ \delta)\left[n_{1}, \ldots, n_{s}\right]=2 T_{4}^{\prime}+2 T_{5}^{\prime}+2 T_{5}^{\prime}=0,
$$

if $n_{j}=2$ with $j<s$. In the case $n_{s}=2$, the contributions containing $n_{s}-2$ are missing in $\delta \circ S, S \circ \delta$ and $D$. So we get

$$
\begin{aligned}
(D-2 \delta \circ S-2 S \circ \delta)\left[n_{1}, \ldots,\right. & \left.n_{s-1}, 2\right]=2 T_{4}^{\prime}+2 T_{8}^{\prime} \\
& =2 \sum_{1 \leqslant k \leqslant s}(-1)^{s+k+\sum_{m=1}^{k-1} n_{m}}\left[n_{1}, \ldots, n_{k-1}, 1, n_{k}, \ldots, n_{s-1}\right] .
\end{aligned}
$$

A similar argument deals with the case that some $n_{j}=1$.

Lemma 4.2. For every partition $\left[n_{1}, \ldots, n_{s}\right]$ with all $n_{i}$ even, we have

$$
(D-2 \delta \circ S-2 S \circ \delta) \operatorname{Ins}_{t}\left[n_{1}, \ldots, n_{s-1}, 2\right]=2(t+1)(-1)^{t+1} \operatorname{Ins}_{t+1}\left[n_{1}, \ldots, n_{s-1}\right] .
$$

Proof. Take any $I \subset\{1, \ldots, s+t\}$ with $|I|=t+1$. The term $\operatorname{Ins}_{I}\left[n_{1}, \ldots, n_{s-1}\right]$ is created in $(D-2 \delta \circ S-2 S \circ \delta) \operatorname{Ins}_{t}\left[n_{1}, \ldots, n_{s-1}, 2\right]$ when the operator $D-2 \delta \circ S-$ $2 S \circ \delta$ inserts a 1 into the summand

$$
\operatorname{Ins}_{\{j \mid j \in I, j<i\} \cup\{j-1 \mid j \in I, j>i\}}\left[n_{1}, \ldots, n_{s-1}, 2\right]
$$

for any position $i \in I$. The coefficient of the summand $\operatorname{Ins}_{I}\left[n_{1}, \ldots, n_{s-1}\right]$ in $(D-2 \delta \circ S-2 S \circ \delta) \operatorname{Ins}_{t}\left[n_{1}, \ldots, n_{s-1}, 2\right]$ is

$$
\begin{aligned}
2(-1)^{s t+(s+t)} \sum_{i \in I}(-1)^{i+\sum_{j \in I, j<i} 1+\sum_{j \in I, j<i} j+\sum_{j \in I, j>i}(j-1)} & \\
= & =2(-1)^{s(t+1)}(t+1)(-1)^{\sum_{j \in I} j} .
\end{aligned}
$$

The contributions in the exponent are an st from $\operatorname{Ins}_{t},(s+t)+i+\sum_{j \in I, j<i} 1$ from $(D-2 \delta \circ S-2 S \circ \delta)$ and $\sum_{j \in I, j<i} j+\sum_{j \in I, j>i}(j-1)$ from $\operatorname{Ins}_{\{j \mid j \in I, j<i\} \cup\{j-1 \mid j \in I, j>i\}}$. Altogether, this is the coefficient of $\operatorname{Ins}_{I}\left[n_{1}, \ldots, n_{s-1}\right]$ in

$$
2(t+1)(-1)^{t+1} \operatorname{Ins}_{t+1}\left[n_{1}, \ldots, n_{s-1}\right] .
$$

Corollary 4.3. Let $p>2$. Define the operator $E=D-2 \delta \circ S-2 S \circ \delta$. Take $a$ monomial $x_{1}^{c_{1}} \cdots x_{k}^{c_{k}} y_{1}^{d_{1}} \cdots y_{l}^{d_{l}} y_{0}$ with size $m$. Then

$$
E\left(x_{1}^{c_{1}} \cdots x_{k}^{c_{k}} y_{1}^{d_{1}} \cdots y_{l}^{d_{l}}\right)=0
$$


and

$$
E\left(x_{1}^{c_{1}} \cdots x_{k}^{c_{k}} y_{1}^{d_{1}} \cdots y_{l}^{d_{l}} y_{0}\right)=2(-1)^{n-m+1}(n-m+1) x_{1}^{c_{1}} \cdots x_{k}^{c_{k}} y_{1}^{d_{1}} \cdots y_{l}^{d_{l}} .
$$

Proof. All entries in all partitions of $x_{1}^{c_{1}} \cdots x_{k}^{c_{k}} y_{1}^{d_{1}} \cdots y_{l}^{d_{l}}$ are different from 2, so by Lemma 4.1 we have $E\left(x_{1}^{c_{1}} \cdots x_{k}^{c_{k}} y_{1}^{d_{1}} \cdots y_{l}^{d_{l}}\right)=0$.

The chain $x_{1}^{c_{1}} \cdots x_{k}^{c_{k}} y_{1}^{d_{1}} \cdots y_{l}^{d_{l}} y_{0}$ can be written as

$$
\operatorname{Ins}_{n-m} \operatorname{Perm}\left[2 p^{i_{1}-1}, 2 p^{i_{1}-1}(p-1), \ldots, 2 p^{i_{k}-1}, 2 p^{i_{k}-1}(p-1), 2 p^{j_{1}}, \ldots, 2 p^{j_{l}}, 2\right]
$$

for some indices $i_{1}, \ldots, i_{k}, j_{1}, \ldots, j_{l}$. By Lemmas 4.1 and 4.2 , the operator $E$ cancels all partitions not having a 2 as last entry, otherwise it removes the last entry. So $E\left(x_{1}^{c_{1}} \cdots x_{k}^{c_{k}} y_{1}^{d_{1}} \cdots y_{l}^{d_{l}} y_{0}\right)$ is given by

$$
2(n+m-1)(-1)^{n+m-1} \operatorname{Ins}_{n-m+1} \operatorname{Perm}\left[2 p^{i_{1}-1}, 2 p^{i_{1}-1}(p-1), \ldots, 2 p^{j_{1}}, \ldots, 2 p^{j_{l}}\right] .
$$

A similar proof deals with the case $p=2$.

Corollary 4.4. Let $p=2$. Take $x_{1}^{c_{1}} \cdots x_{k}^{c_{k}} y_{1}^{d_{1}} \cdots y_{l}^{d_{l}} y_{0}$ with size $m$. Then

$$
E\left(x_{2}^{c_{2}} \cdots x_{k}^{c_{k}} y_{1}^{d_{1}} \cdots y_{l}^{d_{l}}\right)=0
$$

and if $c_{1}>0$

$$
E\left(x_{1}^{c_{1}} \cdots x_{k}^{c_{k}} y_{1}^{d_{1}} \cdots y_{l}^{d_{l}}\right)=2(-1)^{n-m+3}(n-m+3) x_{1}^{c_{1}-1} \cdots x_{k}^{c_{k}} y_{1}^{d_{1}} \cdots y_{l}^{d_{l}} y_{0} .
$$

Furthermore,

$$
E\left(x_{1}^{c_{1}} \cdots x_{k}^{c_{k}} y_{1}^{d_{1}} \cdots y_{l}^{d_{l}} y_{0}\right)=2(-1)^{n-m+1}(n-m+1) x_{1}^{c_{1}} \cdots x_{k}^{c_{k}} y_{1}^{d_{1}} \cdots y_{l}^{d_{l}} .
$$

This allows us to compute the map $D^{*}: H^{i}\left(A_{n}^{\bullet}\right) \rightarrow H^{i-1}\left(A_{n-1}^{\bullet}\right)$ with both $\mathbb{Z}$ and $\mathbb{Z}_{p}$-coefficients.

\section{Proof of main theorem}

Proof of Theorem 1.1. By Corollary 4.3 we can conclude that the rank of the map

$$
D^{*}: H^{r}\left(A_{n}^{\bullet}, \mathbb{Z}_{p}\right) \rightarrow H^{r-1}\left(A_{n-1}^{\bullet}, \mathbb{Z}_{p}\right)
$$

is given by the number of monomials

$$
x_{1}^{c_{1}} \ldots x_{k}^{c_{k}} y_{0} y_{1}^{d_{1}} \ldots y_{l}^{d_{l}}
$$

of degree $r$ and size $m \leqslant n$ such that $p \nmid 2(n-m+1)$. Equivalently, the rank is $B_{p}^{\prime}(n, r)$. By the long exact sequence of Lemma 3.2 we have determined

$$
\operatorname{dim} H^{r}\left(C_{n}\left(S^{2}\right), \mathbb{Z}_{p}\right)=B_{p}(n, r)+B_{p}(n-1, r-2)-B_{p}^{\prime}(n, r)-B_{p}^{\prime}(n, r-1) .
$$

Remark 5.1 (Paolo Salvatore, personal communication). Let

$$
Q=\prod_{i>0} \frac{1+w^{2 p^{i}-1} z^{2 p^{i}}}{1-w^{2 p^{i}-2} z^{2 p^{i}}}
$$

Then we have for $p>2$ :

$$
\sum_{r, n \geqslant 0} \operatorname{dim} H^{r}\left(C_{n}\left(S^{2}\right), \mathbb{Z}_{p}\right) w^{r} z^{n}=\left(\frac{1}{1-z}+\frac{w z^{p+1}}{1-z^{p}}+\frac{w^{3} z^{3}}{1-z}+\frac{w^{2} z}{1-z^{p}}\right) Q .
$$


Corollary 5.2. The groups $H^{r}\left(C_{n}\left(S^{2}\right), \mathbb{Z}_{p}\right)$ are eventually periodic:

$$
\operatorname{dim} H^{r}\left(C_{n+p}\left(S^{2}\right), \mathbb{Z}_{p}\right)=\operatorname{dim} H^{r}\left(C_{n}\left(S^{2}\right), \mathbb{Z}_{p}\right)
$$

if $n \geqslant 2 r+2$.

This is a special case of the general results of [17].

Proof. As $\sum_{i=1}^{g} p^{a_{i}}+\sum_{j=1}^{h} p^{b_{j}} \geqslant 2 g+h$, we get the inequalities $r \geqslant 2 g+h$ and

$$
2 \sum_{i=1}^{g} p^{a_{i}}+2 \sum_{j=1}^{h} p^{b_{j}} \leqslant 2 r .
$$

Hence we have for $n \geqslant 2 r+2$ that

$$
2 \sum_{i=1}^{g} p^{a_{i}}+2 \sum_{j=1}^{h} p^{b_{j}}+2 \leqslant n .
$$

Hence

$$
B_{p}(n, r)=B_{p}(n+1, r), \quad B_{p}^{\prime}(n+p, r)=B_{p}^{\prime}(n, r) .
$$

Proof of Theorem 1.3. For $n \leqslant 3$, we can easily check the theorem by direct computation with $A_{n}^{\bullet}$. Take $n \geqslant 4$. We look at the beginning of the long exact sequence of Lemma 3.2. We immediately read off

$$
H^{0}\left(B_{n}^{\bullet}\right) \simeq H^{0}\left(A_{n}^{\bullet}\right) \simeq \mathbb{Z}
$$

since both spaces are connected. As $H^{2}\left(A_{n}^{\bullet}\right)=H^{2}\left(A_{n-1}^{\bullet}\right)=0$ by application of Theorem 2.13, we get the exact sequence

$$
0 \rightarrow H^{1}\left(B_{n}^{\bullet}\right) \rightarrow H^{1}\left(A_{n}^{\bullet} \stackrel{D^{*}}{\longrightarrow} H^{0}\left(A_{n-1}\right) \rightarrow H^{2}\left(B_{n}^{\bullet}\right) \rightarrow 0 .\right.
$$

The group $H^{1}\left(A_{n}^{\bullet}\right)=\mathbb{Z}$ is generated by the class of $y_{0}$ and the group $H^{0}\left(A_{n-1}^{\bullet}\right)=\mathbb{Z}$ is generated by the class 1 with the map $D^{*}\left(y_{0}\right)=(2 n-2) \cdot 1$ by Corollary 4.3 . Hence we see

$$
H^{1}\left(B_{n}^{\bullet}\right)=0, \quad H^{2}\left(B_{n}^{\bullet}\right)=\mathbb{Z} /(2 n-2) \mathbb{Z} .
$$

If we had $D=2 \delta \circ S+2 S \circ \delta$, we would have a chain map

$$
A_{n}^{\bullet} \rightarrow B_{n}^{\bullet}, a \mapsto\left(a,-2(-1)^{n-r} S(a)\right),
$$

that would split the sequence

$$
0 \rightarrow A_{n-1}^{\bullet}[2] \rightarrow B_{n}^{\bullet} \rightarrow A_{n}^{\bullet} \rightarrow 0, a_{2} \mapsto\left(0, a_{2}\right),\left(a_{1}, a_{2}\right) \mapsto a_{1}
$$

on the right.

In our case, the long exact sequence of Lemma 3.2 gives us short exact sequences

$$
0 \rightarrow \text { Coker } D^{*} \rightarrow H^{r}\left(B_{n}^{\bullet}\right) \rightarrow \operatorname{Ker} D^{*} \rightarrow 0 .
$$

We want to construct a right splitting $s: \operatorname{Ker} D^{*} \rightarrow H^{r}\left(B_{n}^{\bullet}\right)$. For $r \geqslant 2$, the cohomology group $H^{r}\left(A_{n}^{\bullet}\right)$ is finite and has no elements of order $p^{2}$. For every prime $p$, we can take a $\mathbb{Z}_{p}$-basis of the $p$-torsion in Ker $D^{*}$ consisting of the classes $\overline{b_{i}}$ of the chains 


$$
b_{i}=\frac{1}{p} \delta\left(m_{i}\right)
$$

such that $\overline{b_{i}}=\tilde{\beta}\left(m_{i}\right)$ for some monomials $m_{i}=x_{1}^{a_{1}} \ldots x_{k}^{a_{k}} y_{1}^{b_{1}} \ldots y_{l}^{b_{l}} y_{0}^{b_{0}} \in A_{n}^{\bullet}$. By Corollaries 4.3 and 4.4, there are integers $k_{i}^{\prime}$ and monomials $m_{i}^{\prime}$ such that

$$
(D-2 S \circ \delta-2 \delta \circ S)\left(m_{i}\right)=k_{i}^{\prime} m_{i}^{\prime} .
$$

As $b_{i} \in \operatorname{Ker} D^{*}$, we see that $p \mid p_{i}$. Write

$$
(D-2 \circ \delta-2 S \delta \circ S)\left(m_{i}\right)=k_{i} p m_{i}^{\prime} .
$$

Define $E=D-2 S \circ \delta-2 \delta \circ S$. Observe that $E \circ \delta=\delta \circ E$. Hence we get

$$
E\left(m_{i}\right)=p k_{i} m_{i}^{\prime}, \quad E\left(b_{i}\right)=k_{i} \delta\left(m_{i}^{\prime}\right) .
$$

Define a map

$$
s: \operatorname{Ker} D^{*} \rightarrow H^{r}\left(B_{n}^{\bullet}, \mathbb{Z}\right)
$$

by setting

$$
s\left(\overline{b_{i}}\right)=\left(b_{i},-2(-1)^{n-r} S\left(b_{i}\right)-(-1)^{n-r} k_{i} m_{i}^{\prime}\right) .
$$

We see that

$$
\begin{aligned}
\Delta \circ s\left(\overline{b_{i}}\right) & =\left(\delta\left(b_{i}\right),-2(-1)^{n-r} \delta \circ S\left(b_{i}\right)+(-1)^{n-r} D\left(b_{i}\right)-(-1)^{n-r} k_{i} \delta\left(m_{i}^{\prime}\right)\right) \\
& =\left(\delta\left(b_{i}\right), 2(-1)^{n-r} S \circ \delta\left(b_{i}\right)+(-1)^{n-r} E\left(b_{i}\right)-(-1)^{n-r} k_{i} \delta\left(m_{i}^{\prime}\right)\right) \\
& =0
\end{aligned}
$$

and hence $s\left(\bar{b}_{i}\right)$ is a cycle in $H^{r}\left(B_{n}^{\bullet}, \mathbb{Z}\right)$. We have to show that $p s\left(\bar{b}_{i}\right)$ is a boundary. We have $p b_{i}=\delta\left(m_{i}\right)$ and can compute

$$
\begin{aligned}
p s\left(\bar{b}_{i}\right) & =\left(p b_{i},-2(-1)^{n-r} S\left(p b_{i}\right)-(-1)^{n-r} p k_{i} m_{i}^{\prime}\right) \\
& =\left(\delta\left(m_{i}\right),-2(-1)^{n-r} S \circ \delta\left(m_{i}\right)-(-1)^{n-r} p k_{i} m_{i}^{\prime}\right) \\
& =\left(\delta\left(m_{i}\right),(-1)^{n-r}\left(2 \delta \circ S\left(m_{i}\right)-D\left(m_{i}\right)+E\left(m_{i}\right)-p k_{i} m_{i}^{\prime}\right)\right) \\
& =\left(\delta\left(m_{i}\right), 2(-1)^{n-r} \delta \circ S\left(m_{i}\right)-(-1)^{n-r} D\left(m_{i}\right)\right) \\
& =\Delta\left(m_{i}, S\left(m_{i}\right)\right) .
\end{aligned}
$$

Hence $s$ is a well-defined right splitting of the sequence

$$
0 \rightarrow \operatorname{Coker} D^{*} \rightarrow H^{r}\left(B_{n}^{\bullet}\right) \rightarrow \operatorname{Ker} D^{*} \rightarrow 0 .
$$

For $r \geqslant 3$, both $\operatorname{Ker} D^{*}$ and Coker $D^{*}$ have no elements of $p^{2}$, thus the same is true for $H^{r}\left(B_{n}^{\bullet}\right)$.

Example 5.3. We want to compute the 3 -torsion in the groups $H^{6}\left(C_{9}\left(S^{2}\right), \mathbb{Z}\right)$ and $H^{6}\left(C_{10}\left(S^{2}\right), \mathbb{Z}\right)$. We use the long exact sequence

$$
\cdots \rightarrow H^{5}\left(A_{n}^{\bullet}\right) \stackrel{D^{*}}{\longrightarrow} H^{4}\left(A_{n-1}^{\bullet}\right) \rightarrow H^{6}\left(B_{n}^{\bullet}\right) \rightarrow H^{6}\left(A_{n}^{\bullet}\right) \stackrel{D^{*}}{\longrightarrow} H^{5}\left(A_{n-1}^{\bullet}\right) \rightarrow \cdots
$$

for $n=9$ and $n=10$.

For $p=3$, the generators of $H^{*}\left(A_{n}^{\bullet}, \mathbb{Z}_{3}\right)$ are:

\begin{tabular}{c|cccccc} 
generator & $x_{1}$ & $x_{2}$ & $y_{0}$ & $y_{1}$ & $y_{2}$ & $\ldots$ \\
\hline degree & 4 & 16 & 1 & 5 & 17 & $\ldots$ \\
size & 6 & 18 & 2 & 6 & 18 & $\ldots$
\end{tabular}


So

$$
H^{6}\left(A_{9}^{\bullet}, \mathbb{Z}_{3}\right)=H^{6}\left(A_{10}^{\bullet}, \mathbb{Z}_{3}\right)=\mathbb{Z}_{3} y_{0} y_{1}
$$

and

$$
H^{4}\left(A_{9}^{\bullet}, \mathbb{Z}_{3}\right)=H^{4}\left(A_{10}^{\bullet}, \mathbb{Z}_{3}\right)=\mathbb{Z}_{3} x_{1} .
$$

We have $D^{*}\left(y_{0} y_{1}\right)=2(n-7) y_{1}$ and $D^{*}\left(x_{1} y_{0}\right)=2(n-7) x_{1}$. Hence we get

$$
H^{6}\left(B_{9}^{\bullet}, \mathbb{Z}_{3}\right)=0, \quad H^{6}\left(B_{10}^{\bullet}, \mathbb{Z}_{3}\right)=\mathbb{Z}_{3}^{2} .
$$

The Bockstein $\tilde{\beta}\left(x_{1} y_{0}\right)=y_{0} y_{1}$ shows

$$
H^{6}\left(A_{9}^{\bullet}, \mathbb{Z}\right)_{T_{3}}=H^{6}\left(A_{10}^{\bullet}, \mathbb{Z}\right)_{T_{3}}=\mathbb{Z}_{3} y_{0} y_{1}
$$

and

$$
H^{4}\left(A_{9}^{\bullet}, \mathbb{Z}\right)_{T_{3}}=H^{4}\left(A_{10}^{\bullet}, \mathbb{Z}\right)_{T_{3}}=0 .
$$

We get

$$
H^{6}\left(B_{9}^{\bullet}, \mathbb{Z}\right)_{T_{3}}=0, \quad H^{6}\left(B_{10}^{\bullet}, \mathbb{Z}\right)_{T_{3}}=\mathbb{Z}_{3} .
$$

Example 5.4. Tables 3 and 4 were computed with the help of the computer algebra systems Sage [20] and Magma [3]. The integral cohomology groups $H^{r}\left(C_{n}\left(S^{2}\right), \mathbb{Z}\right)$ have already been determined for $n \leqslant 9$ by Sevryuk [23] and Napolitano [18].

Remark 5.5. The whole argument of this paper is very specifically built for $S^{2}$. Similar cell structures exist for other surfaces [18]. However, a more conceptual argument might be useful for these, more complex cases.

\section{References}

[1] V. Arnold. The cohomology ring of the colored braid group. In Vladimir $I$. Arnold-Collected Works, pages 183-186. Springer, 1969.

[2] V. Arnold. On some topological invariants of algebraic functions. In Vladimir I. Arnold-Collected Works, pages 199-221. Springer, 1970.

[3] W. Bosma, J. Cannon, and C. Playoust. The Magma algebra system. I. The user language. J. Symbolic Comput., 24(3-4):235-265, 1997. Computational algebra and number theory (London, 1993).

[4] C. Büdigheimer, F. Cohen, and L. Taylor. On the homology of configuration spaces. Topology, 28:111-123, 1989.

[5] F. Cantero and M. Palmer. On homological stability for configuration spaces on closed background manifolds. Doc. Math., 20:753-805, 2015.

[6] N. Chriss and V. Ginzburg. Representation theory and complex geometry. Mod. Birkhäuser Class. Birkhäuser Boston, Inc., Boston, MA, 2010. Reprint of the 1997 edition.

[7] T. Church. Homological stability for configuration spaces of manifolds. Invent. Math., 188(2):465-504, 2012.

[8] F. Cohen, T. Lada, and P. May. The homology of iterated loop spaces, Lecture Notes in Mathematics, volume 533. Springer-Verlag, 1976. 
Table 3: Cohomology groups $H^{i}\left(C_{n}(\mathbb{C}), \mathbb{Z}\right)$

\begin{tabular}{|c|c|c|c|c|c|c|c|c|c|c|c|c|c|c|c|c|}
\hline$n^{i}$ & 0 & 1 & 2 & 3 & 4 & 5 & 6 & 7 & 8 & 9 & 10 & 11 & 12 & 13 & 14 & 15 \\
\hline 1 & $\mathbb{Z}$ & & & & & & & & & & & & & & & \\
\hline 2,3 & $\mathbb{Z}$ & $\mathbb{Z}$ & & & & & & & & & & & & & & \\
\hline 4,5 & $\mathbb{Z}$ & $\mathbb{Z}$ & 0 & $\mathbb{Z}_{2}$ & & & & & & & & & & & & \\
\hline 6,7 & $\mathbb{Z}$ & $\mathbb{Z}$ & 0 & $\mathbb{Z}_{2}$ & $\mathbb{Z}_{2}$ & $\mathbb{Z}_{3}$ & & & & & & & & & & \\
\hline 8,9 & $\mathbb{Z}$ & $\mathbb{Z}$ & 0 & $\mathbb{Z}_{2}$ & $\mathbb{Z}_{2}$ & $\mathbb{Z}_{6}$ & $\mathbb{Z}_{3}$ & $\mathbb{Z}_{2}$ & & & & & & & & \\
\hline 10,11 & $\mathbb{Z}$ & $\mathbb{Z}$ & 0 & $\mathbb{Z}_{2}$ & $\mathbb{Z}_{2}$ & $\mathbb{Z}_{6}$ & $\mathbb{Z}_{6}$ & $\mathbb{Z}_{2}$ & $\mathbb{Z}_{2}$ & $\mathbb{Z}_{5}$ & & & & & & \\
\hline 12,13 & $\mathbb{Z}$ & $\mathbb{Z}$ & 0 & $\mathbb{Z}_{2}$ & $\mathbb{Z}_{2}$ & $\mathbb{Z}_{6}$ & $\mathbb{Z}_{6}$ & $\mathbb{Z}_{2}^{2}$ & $\mathbb{Z}_{2}$ & $\mathbb{Z}_{2} \times \mathbb{Z}_{3} \times \mathbb{Z}_{5}$ & $\mathbb{Z}_{2} \times \mathbb{Z}_{5}$ & & & & & \\
\hline 14,15 & $\mathbb{Z}$ & $\overline{\mathbb{Z}}$ & 0 & $\mathbb{Z}_{2}$ & $\mathbb{Z}_{2}$ & $\mathbb{Z}_{6}$ & $\mathbb{Z}_{6}$ & $\mathbb{Z}_{2}^{2}$ & $\mathbb{Z}_{2}^{2}$ & $\mathbb{Z}_{2 \times \mathbb{Z}_{3} \times \mathbb{Z}_{5}}$ & $\mathbb{Z}_{2}^{2} \times \mathbb{Z}_{3} \times \mathbb{Z}_{5}$ & $\mathbb{Z}_{2}$ & 0 & $\mathbb{Z}_{7}$ & & \\
\hline 16,17 & $\mathbb{Z}$ & $\bar{Z}$ & 0 & $\mathbb{Z}_{2}$ & $\mathbb{Z}_{2}$ & $\mathbb{Z}_{6}$ & $\mathbb{Z}_{6}$ & $\mathbb{Z}_{2}^{2}$ & $\mathbb{Z}_{2}^{2}$ & $\mathbb{Z}_{2}^{2} \times \mathbb{Z}_{3} \times \mathbb{Z}_{5}$ & $\mathbb{Z}_{2}^{2} \times \mathbb{Z}_{3} \times \mathbb{Z}_{5}$ & $\mathbb{Z}_{2}^{2}$ & $\mathbb{Z}_{2}$ & $\mathbb{Z}_{2} \times \mathbb{Z}_{7}$ & $\mathbb{Z}_{7}$ & $\mathbb{Z}_{2}$ \\
\hline
\end{tabular}

Table 4: Cohomology groups $H^{i}\left(C_{n}\left(S^{2}\right), \mathbb{Z}\right)$

\begin{tabular}{|c|c|c|c|c|c|c|c|c|c|c|c|c|c|c|c|c|}
\hline$n$ & 0 & 1 & 2 & 3 & 4 & 5 & 6 & 7 & 8 & 9 & 10 & 11 & 12 & 13 & 14 & 15 \\
\hline 1 & $\mathbb{Z}$ & 0 & $\mathbb{Z}$ & & & & & & & & & & & & & \\
\hline 2 & $\mathbb{Z}$ & 0 & $\mathbb{Z}_{2}$ & & & & & & & & & & & & & \\
\hline 3 & $\mathbb{Z}$ & 0 & $\mathbb{Z}_{4}$ & $\mathbb{Z}$ & & & & & & & & & & & & \\
\hline 4 & $\mathbb{Z}$ & 0 & $\mathbb{Z}_{6}$ & $\mathbb{Z} \times \mathbb{Z}_{2}$ & & & & & & & & & & & & \\
\hline 5 & $\mathbb{Z}$ & 0 & $\mathbb{Z}_{8}$ & $\mathbb{Z} \times \mathbb{Z}_{2}$ & 0 & $\mathbb{Z}_{2}$ & & & & & & & & & & \\
\hline 6 & $\mathbb{Z}$ & 0 & $\mathbb{Z}_{10}$ & $\mathbb{Z}_{\times} \mathbb{Z}_{2}$ & $\mathbb{Z}_{2}$ & $\mathbb{Z}_{2 \times \mathbb{Z}_{3}}$ & & & & & & & & & & \\
\hline 7 & $\mathbb{Z}$ & 0 & $\mathbb{Z}_{12}$ & $\mathbb{Z}_{\times} \mathbb{Z}_{2}$ & $\mathbb{Z}_{2}$ & $\mathbb{Z}_{2 \times \mathbb{Z}_{3}}$ & $\mathbb{Z}_{2}$ & $\mathbb{Z}_{3}$ & & & & & & & & \\
\hline 8 & $\mathbb{Z}$ & 0 & $\mathbb{Z}_{14}$ & $\mathbb{Z}_{\times} \mathbb{Z}_{2}$ & $\mathbb{Z}_{2}$ & $\mathbb{Z}_{2}^{2} \times \mathbb{Z}_{3}$ & $\mathbb{Z}_{2}$ & $\mathbb{Z}_{2}$ & & & & & & & & \\
\hline 9 & $\mathbb{Z}$ & 0 & $\mathbb{Z}_{16}$ & $\mathbb{Z}_{\times} \mathbb{Z}_{2}$ & $\mathbb{Z}_{2}$ & $\mathbb{Z}_{2}^{2} \times \mathbb{Z}_{3}$ & $\mathbb{Z}_{2}$ & $\mathbb{Z}_{2}^{2}$ & $\mathbb{Z}_{3}$ & $\mathbb{Z}_{2}$ & & & & & & \\
\hline 10 & $\mathbb{Z}$ & 0 & $\mathbb{Z}_{18}$ & $\mathbb{Z}_{\times} \mathbb{Z}_{2}$ & $\mathbb{Z}_{2}$ & $\mathbb{Z}_{2}^{2} \times \mathbb{Z}_{3}$ & $\mathbb{Z}_{2}^{2} \times \mathbb{Z}_{3}$ & $\mathbb{Z}_{2}^{2} \times \mathbb{Z}_{3}$ & $\mathbb{Z}_{2 \times \mathbb{Z}_{3}}$ & $\mathbb{Z}_{2} \times \mathbb{Z}_{5}$ & & & & & & \\
\hline 11 & $\mathbb{Z}$ & 0 & $\mathbb{Z}_{20}$ & $\mathbb{Z} \times \mathbb{Z}_{2}$ & $\mathbb{Z}_{2}$ & $\mathbb{Z}_{2}^{2} \times \mathbb{Z}_{3}$ & $\mathbb{Z}_{2}^{2}$ & $\mathbb{Z}_{2}^{2}$ & $\mathbb{Z}_{2}^{2} \times \mathbb{Z}_{3}$ & $\mathbb{Z}_{2 \times \mathbb{Z}_{5}}$ & $\mathbb{Z}_{2}$ & $\mathbb{Z}_{5}$ & & & & \\
\hline 12 & $\mathbb{Z}$ & 0 & $\mathbb{Z}_{22}$ & $\mathbb{Z}_{\times} \mathbb{Z}_{2}$ & $\mathbb{Z}_{2}$ & $\mathbb{Z}_{2}^{2} \times \mathbb{Z}_{3}$ & $\mathbb{Z}_{2}^{2}$ & $\mathbb{Z}_{2}^{3}$ & $\mathbb{Z}_{2}^{2} \times \mathbb{Z}_{3}$ & $\mathbb{Z}_{2}^{2} \times \mathbb{Z}_{3} \times \mathbb{Z}_{5}$ & $\mathbb{Z}_{2}^{2}$ & 0 & & & & \\
\hline 13 & $\mathbb{Z}$ & 0 & $\mathbb{Z}_{24}$ & $\mathbb{Z}_{\times} \mathbb{Z}_{2}$ & $\mathbb{Z}_{2}$ & $\mathbb{Z}_{2}^{2} \times \mathbb{Z}_{3}$ & $\mathbb{Z}_{2}^{2} \times \mathbb{Z}_{3}$ & $\mathbb{Z}_{2}^{3} \times \mathbb{Z}_{3}$ & $\mathbb{Z}_{2}^{2} \times \mathbb{Z}_{3}$ & $\mathbb{Z}_{2}^{3} \times \mathbb{Z}_{3} \times \mathbb{Z}_{5}$ & $\mathbb{Z}_{2}^{2}$ & $\mathbb{Z}_{2} \times \mathbb{Z}_{3}$ & $\mathbb{Z}_{2} \times \mathbb{Z}_{5}$ & & & \\
\hline 14 & $\mathbb{Z}$ & 0 & $\mathbb{Z}_{26}$ & $\mathbb{Z}_{\times} \mathbb{Z}_{2}$ & $\mathbb{Z}_{2}$ & $\mathbb{Z}_{2}^{2} \times \mathbb{Z}_{3}$ & $\mathbb{Z}_{2}^{2}$ & $\mathbb{Z}_{2}^{3}$ & $\mathbb{Z}_{2}^{3} \times \mathbb{Z}_{3}$ & $\mathbb{Z}_{2}^{3} \times \mathbb{Z}_{3} \times \mathbb{Z}_{5}$ & $\mathbb{Z}_{2}^{3}$ & $\mathbb{Z}_{2}^{2}$ & $\mathbb{Z}_{2 \times \mathbb{Z}_{5}}$ & $\mathbb{Z}_{7}$ & & \\
\hline 15 & $\mathbb{Z}$ & 0 & $\mathbb{Z}_{28}$ & $\mathbb{Z} \times \mathbb{Z}_{2}$ & $\mathbb{Z}_{2}$ & $\mathbb{Z}_{2}^{2} \times \mathbb{Z}_{3}$ & $\mathbb{Z}_{2}^{2}$ & $\mathbb{Z}_{2}^{3}$ & $\mathbb{Z}_{2}^{3} \times \mathbb{Z}_{3}$ & $\mathbb{Z}_{2}^{3} \times \mathbb{Z}_{3} \times \mathbb{Z}_{5}$ & $\mathbb{Z}_{2}^{4}$ & $\mathbb{Z}_{2}^{2}$ & $\mathbb{Z}_{2}^{2} \times \mathbb{Z}_{3} \times \mathbb{Z}_{5}$ & $\mathbb{Z}_{2 \times} \times \mathbb{Z}_{7}$ & 0 & $\mathbb{Z}_{7}$ \\
\hline 16 & $\mathbb{Z}$ & 0 & $\mathbb{Z}_{30}$ & $\mathbb{Z} \times \mathbb{Z}_{2}$ & $\mathbb{Z}_{2}$ & $\mathbb{Z}_{2}^{2} \times \mathbb{Z}_{3}$ & $\mathbb{Z}_{2}^{2} \times \mathbb{Z}_{3}$ & $\mathbb{Z}_{2}^{3} \times \mathbb{Z}_{3}$ & $\mathbb{Z}_{2}^{3} \times \mathbb{Z}_{3}$ & $\mathbb{Z}_{2}^{4} \times \mathbb{Z}_{3} \times \mathbb{Z}_{5}$ & $\mathbb{Z}_{2}^{4} \times \mathbb{Z}_{3} \times \mathbb{Z}_{5}$ & $\mathbb{Z}_{2}^{3} \times \mathbb{Z}_{3} \times \mathbb{Z}_{5}$ & $\mathbb{Z}_{2}^{3} \times \mathbb{Z}_{3} \times \mathbb{Z}_{5}$ & $\mathbb{Z}_{2}^{2} \times \mathbb{Z}_{7}$ & 0 & $\mathbb{Z}_{2}$ \\
\hline
\end{tabular}


[9] G. Drummond-Cole and B. Knudsen. Betti numbers of configuration spaces of surfaces. ArXiv e-prints, August 2016.

[10] E. Fadell and S. Husseini. Geometry and topology of configuration spaces. Springer, 2001.

[11] Y. Felix, S. Halperin, and J. Thomas. Rational homotopy theory, Graduate Texts in Mathematics, 205 Springer-Verlag, New York, 2001.

[12] N.J. Fine. Binomial coefficients modulo a prime. Amer. Math. Monthly, 54:589$592,1947$.

[13] R. Fox and L. Neuwirth. The braid groups. Math. Scand., 10:119-126, 1962.

[14] D.B. Fuks. Cohomology of the braid group $\bmod 2$. Funktsional. Anal. $i$ Prilozhen., 4(2):62-73, 1970.

[15] A. Hatcher. Algebraic topology. Cambridge University Press, 2002.

[16] A. Kupers and J. Miller. Sharper periodicity and stabilization maps for configuration spaces of closed manifolds. Proc. Amer. Math. Soc., 144(12):5457-5468, 2016.

[17] R. Nagpal. FI-modules and the cohomology of modular representations of symmetric groups. ProQuest LLC, Ann Arbor, MI, 2015. Thesis (Ph.D.) - The University of Wisconsin-Madison.

[18] F. Napolitano. On the cohomology of configuration spaces on surfaces. J. Lond. Math. Soc. (2), 68:477-492, 2003.

[19] O. Randal-Williams. Topological chiral homology and configuration spaces of spheres. Morfismos, 17(2):57-69, 2013.

[20] Sage Developers. Sage Mathematics Software (Ver. 8.2), 2018. sagemath.org.

[21] P. Salvatore. Configuration spaces on the sphere and higher loop spaces. Math. Z., 248(3):527-540, 2004.

[22] G. Segal. Configuration-spaces and iterated loop-spaces. Invent. Math., 21:213221, 1973.

[23] M. Sevryuk. The cohomology of projectively compactified complex swallowtails and their complements. Russian Math. Surveys, 39(5):285, 1984.

[24] B. Totaro. Configuration spaces of algebraic varieties. Topology, 35(4):10571067, 1996.

[25] F.V. Vainshtein. The cohomology of braid groups. Funktsional. Anal. $i$ Prilozhen., 12(2):72-73, 1978.

[26] V.A. Vassiliev. Introduction to topology, volume 14 of Stud. Math. Libr. American Mathematical Society, Providence, RI, 2001. Translated from the 1997 Russian original by A. Sossinski.

[27] V. Vershinin. Homology of braid groups and their generalizations. In Knot theory (Warsaw, 1995), volume 42 of Banach Center Publ., pages 421-446. Polish Acad. Sci. Inst. Math., Warsaw, 1998.

Christoph Schiessl christoph.schiessl@posteo.ch

ETH Zurich, Lindenstrasse 3, Steinmaur, 8162, Switzerland 